\title{
Perfil bibliométrico de la odontología chilena, 2001-2010
}

\author{
Bibliometric profile of chilean dentistry, 2001-2010
}

Cartes-Velásquez $\mathrm{R}^{1,2}$, Aravena Torres $\mathrm{P}^{2,3}$

\begin{abstract}
RESUMEN
Introducción: Caracterizar la producción científica personal, institucional o nacional es una necesidad creciente para comprender y mejorar la generación de conocimiento. El objetivo de este trabajo es definir el perfil bibliométrico de la odontología chilena en la última década. Metodología: En la plataforma Web of Knowledge, mediante un algoritmo se realizó la búsqueda de las publicaciones odontológicas chilenas ISI. Los datos: número de publicaciones: factor de impacto (FI); Top-10 de: autores, instituciones, revistas y países de coautoría se muestran para el período 2001-2010 y sus quinquenios Resultados: El número total de publicaciones fue de 195, con una media anual de 12.8 y un FI de 1.18 para el primer quinquenio, cifras aumentadas a $26.2(105 \%)$ y $1.82(54 \%)$ respectivamente en el segundo, pero con una disminución del FI en el último bienio a 1.73 . En cuanto a autores destaca Gamonal; en instituciones la UChile seguida desde muy atrás por la UdeC y otras que aumentaron su producción como UFro, UMayor, UValpo y PUC; en cooperación internacional lo hacen España, USA, Brasil y Suecia, pero con porcentajes menores; en revistas destacan los de periodoncia y 2 publicaciones nacionales no odontológicas en el segundo quinquenio. Discusión: Aunque el aumento de la producción es claro durante la última década, aún está muy por debajo de los grandes productores internacionales y preocupa la disminución del impacto en los últimos 2 años, la alta concentración en la producción (UChile) y la baja cooperación internacional, situaciones que deben ser resueltas en pos de mantener el crecimiento.
\end{abstract}

Rev. Clin. Periodoncia Implantol. Rehabil. Oral Vol. 5(1); 5-8, 2012.

Palabras clave: Odontología, Chile, bibliometría, evaluación.

\begin{abstract}
Introduction: There is an increasing need to characterize personal, institutional or national scientific output in order to understand and improve the generation of knowledge. The aim of this paper is to define the bibliometric profile of Chilean dentistry for the last decade. Methodology: A search was performed in WOK platform using an algorithm to define ISI Chilean dental publications. Data: number of publications, impact factor (IF), TOP-10 for: authors, institutions, journals and co-authored countries are shown for the period 2001-2010 and its five-year periods. Results: The total number of publications was 195, with an annual average of 12.8 and an IF of 1.18 for the first five-years, numbers increased to 26.1 (105\%) and 1.82 (54\%) respectively in the second ones, but with an IF decrease to 1.73 for last two-years. Among the authors, Gamonal stands out in institutions the UChile leads, followed far behind by the UdeC and other universities which improved their participation in the second half as UFro, UMayor, UValpo and PUC; in international cooperation Spain, USA, Brazil and Sweden, albeit with lower rates; as for journals, periodontology detaches itself and in the second half two non-dental Chilean publications. Discussion: There is a noticeable rise of production over the last decade, but still very far from big international producers and the IF decrease in the last two-years is a concern, together with the high concentration of production (UChile) and the low rate of international cooperation, facts that should be improved, in order to maintain the growth.
\end{abstract}

Rev. Clin. Periodoncia Implantol. Rehabil. Oral Vol. 5(1); 5-8, 2012.

Key words: Dentistry, Chile, bibliometry, assessment.

\section{INTRODUCCIÓN}

En la actualidad, la producción del conocimiento científico y la generación de un campo comercial (patentes, relación con el sector privado, entre otras) dentro de los centros de investigación hacen imprescindible la necesidad de tener herramientas de medición como son los análisis bibliométricos ${ }^{(1)}$. Éstos, son ejercicios para el eficiente desarrollo de la investigación, permitiendo caracterizar la producción científica de personas e instituciones, tanto en términos cualitativos como cuantitativos, permitiendo reducir al mínimo de la subjetividad inherente a la indexación del conocimiento en un campo determinado de la ciencia ${ }^{(2,3)}$.

Gracias a estas evaluaciones podemos comparar realidades e identificar áreas fuertes/débiles, potenciales/consolidadas, dispersas/ concentradas, con lo que podemos tener una orientación para priorizar investigadores y/o líneas de investigación, mediante la adjudicación de recursos, más allá de la calidad que promete un proyecto o protocolo ${ }^{(1,2)}$.

Las 2 medidas de mayor importancia en estas evaluaciones son las de productividad y el factor de impacto (FI). La productividad es definida como la cantidad de publicaciones y generalmente solo se incluyen a los artículos. El FI fue descrita por primera vez en 1955 en Estados Unidos y corresponde al cuociente entre el número de citas del año en curso a todos los artículos publicados durante los 2 años previos y el número de dichos artículos ${ }^{(4,5)}$. Como una forma de sintetizar estas 2 medidas en un solo número se han diseñado varios índices ${ }^{(6)}$, siendo el más usado el factor $h$, que corresponde al valor de $\mathrm{n}$ publicaciones que tengan al menos $\mathrm{n}$ citas $^{(7)}$, vale decir, un investigador con un factor $\mathrm{h}$ de 13 tiene 13 publicaciones con al menos 13 citas cada una.

Pero no todas las publicaciones ni citaciones son consideradas para estos análisis, actualmente se utilizan principalmente aquellas indexadas en 2 bases de datos especialmente diseñadas para estos fines, siendo la más conocida y utilizada Thomson-Reuters (ex-ISI), aunque en los últimos años ha entrado con fuerza el uso de Scopus (Elsevier) debido principalmente a que es un índice más inclusivo y representativo que IS|(8), situación que ha sido subsanada en algún grado con el proceso de internacionalización que ha llevada Thomson-Reuters desde el $2006^{(9)}$, el que logró indexar entre miles de revistas de carácter regional a varias del ámbito biomédico chileno(10,11).

A pesar de la utilidad de estas evaluaciones, es poco frecuente encontrar publicaciones enfocadas a evaluar la ciencia odontológica ${ }^{(12-16)}$ menos aún en Latinoamérica ${ }^{(17,18)}$ y para el caso de Chile donde solo encontramos presentaciones en congresos ${ }^{(19)}$.

Dado lo anterior se hace necesario contar con datos que nos permitan conocer cuál es la realidad actual de la investigación odontológica en Chile, el carácter de su evolución en los últimos años y sus posibles caminos de desarrollo futuro. El objetivo de la presente investigación es describir el perfil bibliométrico de la odontología chilena entre los años 2001 y 2010.

\section{METODOLOGÍA}

Se realizó un estudio de tipo descriptivo para determinar las características bibliométricas de la odontología chilena, el que tuvo un carácter retrospectivo para la evaluación de la producción y de corte transversal para el impacto. Para ello, se incluyeron sólo las publicaciones indexadas entre los años 2001 a 2010 en la base de datos Science Citation Index Expanded (SCI-E) de Thomson Reuters.

Para la determinación de las publicaciones se utilizó la búsqueda

1. Cirujano Dentista. Centro de I+D en Odontología Social, CIDOS.org. Chile.

2. Cirujano Dentista. Programa de Doctorado en Ciencias Médicas, Universidad de la Frontera. Chile.

3. Cirujano Dentista. Instituto de Odontoestomatología, Facultad de Medicina, Universidad Austral de Chile. Chile. 
avanzada de la plataforma Web of Knowledge (WOK), en julio de 2011, utilizando un filtro por país (Chile), tema (Dentistry, Oral Medicine \& Surgery) y afiliación de los autores (Dentistry u Odontology), tal como se muestra a continuación: (CU=CHILE AND SJ=(DENTISTRY ORAL SURGERY MEDICINE)) OR (AD=(DENT SAME CHILE) OR AD=(ODONTOL SAME CHILE))

El objetivo de aplicar este algoritmo de búsqueda es incluir en el análisis a todas las publicaciones en revistas del tema "Dentistry, Oral Surgery and Medicine" con al menos un autor chileno, sumando a todas las que bajo este y otros temas incluyan al menos un autor con afiliación a una institución odontológica chilena, de esta manera tenemos una visión más amplia e inclusiva de lo que los autores chilenos en el área odontológica están publicando a nivel internacional. Desde los resultados que arrojó la aplicación de este algoritmo se aplicó un filtro para incluir solo las publicaciones tipificadas como Articles.

A partir de estos resultados se realizó un Citation Report y sucesivos Analize Results, en los cuales se determinaron las variables: producción y número de citas por año (impacto), así como la cantidad de publicaciones por investigador, revistas en las que se publica, instituciones, naciones co-autoras y temas de publicación.

Para facilitar un análisis comparativo se realizó un análisis por quinquenios, 2001-2005 y 2006-2010, así como el bienio 2009-2010, con el fin de apreciar de mejor forma la evolución bibliométrica en estos últimos 10 años. La tabulación y presentación de los datos se realizó con el software MS Excel 2003

\section{RESULTADOS}

Para todo el período en estudio se hallaron 195 artículos, con un promedio anual de $19.5 \pm 9.55$ artículos, la distribución por años se aprecia en el Gráfico 1. Para el primer quinquenio la producción media anual fue de $12.8 \pm 5.93$ y en el segundo de $26.2 \pm 7.6$, en el último bienio fue de $31 \pm 1.41$.

Los 195 artículos recibieron un total de 338 citas durante el período estudiado, promedio de 1.73 por artículo. El FI promedio para el primer quinquenio fue de $1.18 \pm 0.3$ y de $1.82 \pm 0.45$ en el segundo, para el último bienio tuvo un valor de $1.73 \pm 0.36$, los valores para cada año se muestran en el Gráfico 2. La ciencia odontológica chilena, para el período estudiado, tuvo un factor $\mathrm{h}$ de 21.

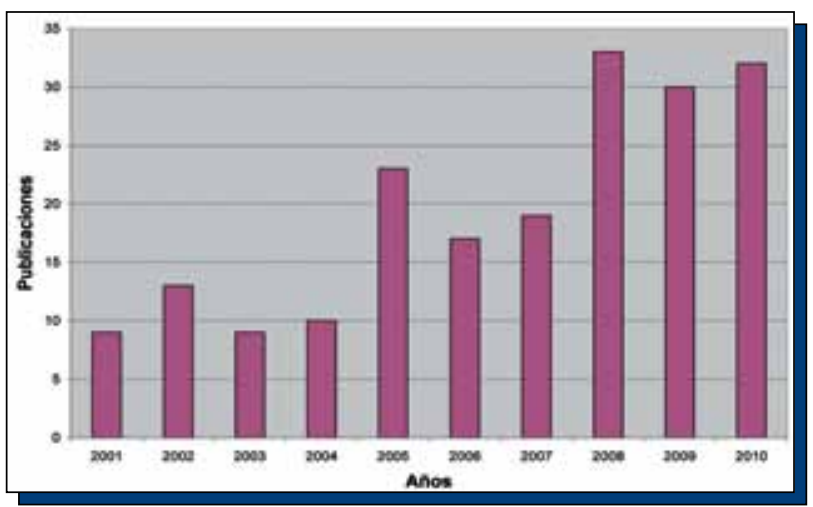

Gráfico 1. Distribución por año de los artículos ISI de la odontología chilena, 2001-2010.

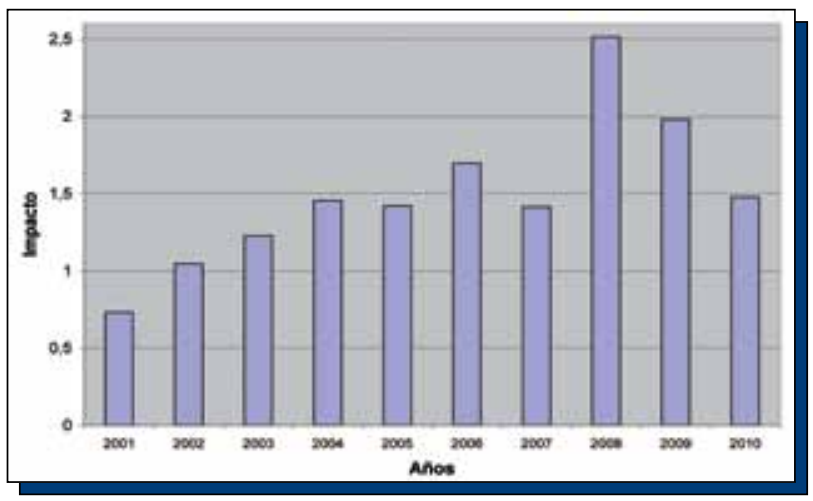

Gráfico 2. Factor de impacto por año de los artículos ISI de la odontología chilena, 2001-2010.
En la Tabla 1 se muestran los artículos más citados para el período en estudio, el número de citas incluye aquellas realizadas hasta julio de 2011. En las Tablas 2 y 3 se muestran respectivamente los autores e instituciones más productivas para el período total, primer quinquenio, segundo quinquenio y último bienio. En la Tabla 4 se exponen las revistas con la mayor cantidad de artículos chilenos publicados para los períodos de tiempo anteriormente mencionados.

Si consideramos los temas (áreas del conocimiento) de las revistas donde se publica, encontramos que para todo el período los más representativos fueron Odontología, Medicina y Cirugía Oral con 133, Anatomía y Morfología con 16, Medicina Interna y General con 11 Patología con 8 , Salud Ocupacional, Ambiental y Pública con 5 y Cirugía también con 5 .

En términos de cooperación internacional la Tabla 5 muestra los países con mayor cantidad de artículos en coautoría.

Solo 46 artículos declararon financiamiento de alguna agencia, las más representativas fueron CONICYT a través de alguno de sus fondos con 15 apariciones y la Universidad de Chile (UChile) con 5

Tabla 1. Artículos ISI de la odontología chilena más citados, 2001-2010.

\begin{tabular}{|l|c|c|}
\hline \multicolumn{1}{|c|}{ Artículo } & $\begin{array}{c}\text { Total de } \\
\text { Citas }\end{array}$ & $\begin{array}{c}\text { Citas } \\
\text { por año }\end{array}$ \\
\hline $\begin{array}{l}\text { Periodontal therapy may reduce the risk of preterm low } \\
\text { birth weight in women with periodontal disease: A rand- } \\
\text { omized controlled trial }\end{array}$ & 160 & 16 \\
\hline $\begin{array}{l}\text { Higher risk of preterm birth and low birth weight in women } \\
\text { with periodontal disease }\end{array}$ & 98 & 9,8 \\
\hline $\begin{array}{l}\text { Guidelines for the management of traumatic dental inju- } \\
\text { ries. II. Avulsion of permanent teeth }\end{array}$ & 73 & 14,6 \\
\hline $\begin{array}{l}\text { Characterization of cellular infiltrate, detection of } \\
\text { chemokine receptor CCR5 and interleukin-8 and } \\
\text { RANTES chemokines in adult periodontitis }\end{array}$ & 60 & 5,45 \\
\hline $\begin{array}{l}\text { Subgingival microbiota of chronic periodontitis subjects } \\
\text { from different geographic locations }\end{array}$ & 55 & 7,38 \\
\hline $\begin{array}{l}\text { Guidelines for the management of traumatic dental inju- } \\
\text { ries. I. Fractures and luxations of permanent teeth }\end{array}$ & 54 & 6,67 \\
\hline $\begin{array}{l}\text { Traumatic injuries in the primary dentition } \\
\text { social gradients in periodontal diseases among adoles- } \\
\text { cents }\end{array}$ & 34 \\
\hline $\begin{array}{l}\text { In situ detection of matrix metalloproteinase-9 (MMP-9) in } \\
\text { gingival epithelium in human periodontal disease } \\
\text { pupernatants of cellular cultures of gingival tissue from }\end{array}$ & 54,43 \\
\hline \hline
\end{tabular}

Tabla 2. Autores según número de artículos ISI por períodos.

\begin{tabular}{|l|c|l|c|l|c|l|c|}
\hline \multicolumn{1}{|c|}{ 2001-2005 } & \multicolumn{2}{c|}{ 2006-2010 } & \multicolumn{2}{c|}{ 2001-2010 } & \multicolumn{2}{c|}{ 2009-2010 } \\
\hline Gutor & N & \multicolumn{1}{c|}{ Autor } & N & \multicolumn{1}{c|}{ Autor } & N & Autor & N \\
\hline Lopez, NJ & 6 & Hernandez, M & 10 & Smith, PC & 14 & Dutzan, N & 6 \\
\hline Jara, L & 5 & Martinez, J & 10 & Vernal, R & 12 & Olate, S & 6 \\
\hline Martinez, B & 5 & Smith, PC & 10 & Leon, R & 11 & Hernandez, M & 5 \\
\hline Miralles, R & 5 & Vernal, R & 10 & Martinez, J & 11 & Martinez, J & 5 \\
\hline Rojas, IG & 4 & Dutzan, N & 9 & Dutzan, N & 10 & Smith, PC & 5 \\
\hline Rudolph, MI & 4 & Caceres, M & 8 & Hernandez, M & 10 & Caceres, M & 4 \\
\hline Smith, PC & 4 & Leon, R & 8 & Miralles, R & 10 & Dezerega, A & 4 \\
\hline Acevedo, A & 3 & Silva, A & 7 & Rojas, IG & 10 & Fernandez, RF & 4 \\
\hline Baelum, V & 3 & Brethauer, U & 6 & Silva, A & 10 & Arancibia, R & 3 \\
\hline
\end{tabular}


Tabla 3. Instituciones según número de artículos ISI por períodos.

\begin{tabular}{|l|c|l|c|l|c|l|l|}
\hline \multicolumn{2}{|c|}{ 2001-2005 } & \multicolumn{2}{c|}{ 2006-2010 } & \multicolumn{2}{c|}{ 2001-2010 } & \multicolumn{2}{c|}{ 2009-2010 } \\
\hline Institución & $\mathbf{N}$ & Institución & $\mathbf{N}$ & Institución & $\mathbf{N}$ & Institución & $\mathbf{N}$ \\
\hline UCHILE & 46 & UCHILE & 71 & UCHILE & 117 & UCHILE & 35 \\
\hline UDEC & 7 & UFRO & 20 & UDEC & 23 & UFRO & 17 \\
\hline U MAYOR & 5 & UDEC & 16 & UFRO & 20 & UDEC & 9 \\
\hline UNIVAAHUS & 3 & PUCCHILE & 8 & PUCCHILE & 11 & UNICAMP & 6 \\
\hline U ANTIQUIA & 3 & UNICAMP & 6 & UMAYOR & 10 & PUCCHILE & 5 \\
\hline PUCCHILE & 3 & UTALCA & 6 & UVALPO & 9 & UANTOF & 3 \\
\hline UCOMPLUT & 3 & UVALPO & 6 & UCOPLUT & 7 & UNIVHESINKI & 3 \\
\hline UVALPO & 3 & UMAYOR & 5 & CSIC & 6 & UTALCA & 3 \\
\hline CESUNIV & 2 & CSIC & 4 & UNICAMP & 6 & & \\
\hline CSIC & 2 & UCOMPLUT & 4 & UWTALCA & 6 & & \\
\hline
\end{tabular}

Tabla 4. Revistas según número de artículos por períodos.

\begin{tabular}{|c|c|c|c|c|c|c|c|}
\hline Journal Of Periodontology & 10 & $\begin{array}{l}\text { International Journal Of } \\
\text { Morphology }\end{array}$ & 16 & Journal Of Periodontology & 20 & $\begin{array}{l}\text { International Journal Of } \\
\text { Morphology }\end{array}$ & 16 \\
\hline $\begin{array}{l}\text { Cranio-The Journal Of Cra- } \\
\text { niomandibular Practice }\end{array}$ & 6 & $\begin{array}{l}\text { Journal Of Periodon- } \\
\text { tology }\end{array}$ & 10 & $\begin{array}{l}\text { International Journal Of } \\
\text { Morphology }\end{array}$ & 16 & Journal Of Periodontology & 6 \\
\hline $\begin{array}{l}\text { Journal Of Oral Pathology } \\
\text { \& Medicine }\end{array}$ & 5 & Dental Traumatology & 7 & $\begin{array}{l}\text { Journal Of Clinical Perio- } \\
\text { dontology }\end{array}$ & 11 & $\begin{array}{l}\text { Medicina Oral Patologia } \\
\text { Oral Y Cirugia Bucal }\end{array}$ & 4 \\
\hline $\begin{array}{l}\text { Journal Of Clinical Perio- } \\
\text { dontology }\end{array}$ & 4 & $\begin{array}{l}\text { Journal Of Periodontal } \\
\text { Research }\end{array}$ & 6 & $\begin{array}{l}\text { Cranio-The Journal Of Cra- } \\
\text { niomandibular Practice }\end{array}$ & 10 & $\begin{array}{l}\text { Journal Of Periodontal } \\
\text { Research }\end{array}$ & 3 \\
\hline $\begin{array}{l}\text { Journal Of Periodontal } \\
\text { Research }\end{array}$ & 4 & $\begin{array}{l}\text { Medicina Oral Patologia } \\
\text { Oral Y Cirugia Bucal }\end{array}$ & 6 & $\begin{array}{l}\text { Journal Of Periodontal } \\
\text { Research }\end{array}$ & 10 & Oral Diseases & 3 \\
\hline
\end{tabular}

Tabla 5. Países en coautoría según número de artículos por períodos.

\begin{tabular}{|l|l|l|l|l|l|l|l|}
\hline \multicolumn{2}{|c|}{ 2001-2005 } & \multicolumn{2}{c|}{ 2006-2010 } & \multicolumn{2}{c|}{ 2001-2010 } & \multicolumn{2}{c|}{ 2009-2010 } \\
\hline \multicolumn{1}{|c|}{ País } & N & \multicolumn{1}{c|}{ País } & $\mathbf{N}$ & \multicolumn{1}{c|}{ País } & N & \multicolumn{1}{c|}{ País } & N \\
\hline USA & 8 & Spain & 15 & Spain & 21 & Brazil & 6 \\
\hline Spain & 6 & Brazil & 6 & USA & 14 & Spain & 6 \\
\hline Colombia & 5 & USA & 6 & Brazil & 10 & Canada & 3 \\
\hline Brazil & 4 & Canada & 5 & Sweden & 8 & England & 3 \\
\hline Denmark & 3 & Sweden & 5 & Colombia & 7 & Finland & 3 \\
\hline Sweden & 3 & Denmark & 4 & Denmark & 7 & $\begin{array}{l}\text { Nether- } \\
\text { lands }\end{array}$ & 3 \\
\hline
\end{tabular}

\section{DISCUSIÓN}

En la última decada, la producción científica odontológica de Chile muestra una tendencia al alza en el número de artículos publicados, duplicándose en los últimos cinco años, y aumentando la calidad (medida a través del FI) de su producción; sin embargo, lo anterior parece haber tocado techo el año 2008 cuando se alcanzó un FI de 2.51 que luego ha bajado en 2009 (1.98) y nuevamente en 2010 (1.48), esto es preocupante ya que denotaría una incipiente pérdida de la calidad de la investigación dental chilena, cuestión que debe ser vigilada durante los próximos años. Entre el año 1999 y 2003 Chile se ubicaba en la posición $39^{\circ}$ del ranking mundial de publicaciones ISI odontológicas, liderando este ranking EE.UU., Reino Unido y Japón, quienes concentran mas del $60 \%$ de la producción total de la muestra ${ }^{(5,16)}$. En Latinoamérica, nuestro país se encontró superado por Brasil $\left(6^{\circ}\right)$ Argentina $\left(34^{\circ}\right)$ y México $\left(38^{\circ}\right)$. Dicha situación debería haber mejorado o al menos mantenido, considerando el crecimiento de la producción, sin embargo, no existen estudios recientes de carácter global que lo confirmen ${ }^{(5)}$.

En términos cualitativos vemos que existe una fuerte concentración de quienes generan las publicaciones, con un núcleo posicionado en la UChile y la investigación en periodoncia; si bien esto es positivo en términos de fortalecer una línea de investigación de alto impacto y visibilidad internacional, también implica que se pueden dar los vicios de mercados monopólicos en que las oportunidades de fortalecer otras líneas o fuentes de investigación se ven disminuidas, una forma de presentación del "efecto Mateo"(20)

Lo anterior queda reflejado en la tímida aparición de otras instituciones al rezago de la UChile en la generación de publicaciones, entre ellas destacan las universidades de Concepción (UdeC), Católica (PUC), de Valparaíso (UValpo) y de la Frontera (UFro), aunque esta última con un alto porcentaje de publicaciones endogámicas, en el International Journal of Morphology, que han generado una bajísima cantidad de citas, esto pone en duda sus niveles de producción científica y podrían ser, en parte, responsables de la baja del FI en el último bienio.

También encontramos una alta concentración en relación a los autores, donde Jorge Gamonal domina ampliamente todo el período, seguido por otros autores afiliados a la UChile y Patricio Smith de la PUC.

Vemos un bajo porcentaje de cooperación internacional y además concentrada en el núcleo previamente mencionado, lo que era esperable dada la relación existente entre productividad, calidad (impacto) e internacionalización de las publicaciones ${ }^{(15,21)}$, por tanto fortalecer la cooperación internacional es una estrategia vital para el crecimiento con calidad de la ciencia odontológica del país.

En términos de financiamiento solo 1 de cada 4 publicaciones declara ser apoyada por algún fondo, sin embargo, esta cifra puede no representar la realidad dado que no siempre se especifican dichas fuentes o son requeridas por las revistas. En nuestro pais, los fondos concursables son transversales a todo tipo de investigador y dependen netamente del campo científico $y$ al monto necesario para el desarrollo del proyecto. Estos fondos se pueden obtener en los mismos centros universitarios y también en instancias gubernamentales como es la Comisión Nacional de Investigación y Tecnología (CONICYT) dependiente del Ministerio de Educación.

El aumento de recursos públicos en investigación permite alcanzar un mayor número de publicaciones en revistas indexadas. Como es el caso de China, donde el 2009 tuvo una tasa de crecimiento en publicaciones de $227 \%$, cifra estrechamente relacionada con el apoyo del gobierno a la investigación científica en la odontología ${ }^{(13)}$, esto refuerza la idea que el crecimiento de la producción en investigación dental va de la mano con lo emergente que sea un país, tal como ha sucedido en Brasil(22).

El amplio déficit de conocimiento que se observa en la mayoría de los países en desarrollo genera una profunda brecha social interna y con los países desarrollados, que no cesó de agravarse desde el salto productivo que representa en la economía mundial el advenimiento de las nuevas tecnologías (informática, robótica, electrónica, biotecnologías, etc.). El gasto nacional en investigación y desarrollo permite hacer una primera aproximación al potencial científico y tecnológico de las naciones. De los 25 mil millones de dólares invertidos por el mundo en 1992 en ciencia y tecnología, el $83 \%$ fue realizado por EE. UU. (38.5\%), la Unión Europea (28.3\%) y Japón (15.8\%). En América Latina representa apenas el 1\% de los gastos mundiales y el $1.4 \%$ de la producción científica mundial|(23).

Vemos que la ciencia odontológica chilena está en un expectante y floreciente período de crecimiento científico con marcadas líneas de investigación. Esto puede deberse a que la valoración de la clínica sobre la base de evidencia ya no se considera una cuestión de filosofía, sino el resultado de la evolución de las ciencias de la salud y, al mismo tiempo, de una disminución en la importancia de los antiguos dogmas, porque es en los científicos donde los profesionales clínicos buscan los estudios pertinentes que proporcionan las respuestas a las peticiones de sus pacientes ${ }^{(3)}$.

El presente trabajo es una primera aproximación formal a la manera en que se hace ciencia odontológica en Chile, donde si bien hallamos un progresivo aumento de la producción en los 
últimos 10 años, este crecimiento no está exento de debilidades como la alta concentración de las fuentes productoras y la baja proporción de cooperación internacional, pero lo que más preocupa es el estancamiento del número de publicaciones en los últimos 3 años y la baja de la calidad (medida por el FI) en 2009 y 2010 ; todas ellas, situaciones que deben seguir siendo vigiladas y enfrentadas con estrategias que permitan mantener un crecimiento con calidad de la producción científica de la odontología chilena.

\section{CONFLICTOS DE INTERÉS}

Los autores declaran no tener conflictos de interés.

\section{REFERENCIAS BIBLIOGRÁFICAS}

1. Baskurt OK. Time series analysis of publication counts of a university: What are the implications? Scientometrics, 2011; 86(3): 645-656.

2. Abramo G, Ciriaco A, Di Costa F. National research assessment exercises: A comparison of peer review and bibliometrics rankings. Scientometrics, 2011. En prensa.

3. Poletto VC, Faraco IM. Bibliometric study of articles published in a Brazilian journal of pediatric dentistry. Braz Oral Res, 2010; 24(1): 83-88.

4. Chattopadhyay A. How useful is journal impact factor? Indian J Dent Res, 2009; 20: 246-248.

5. Gil-Montoya JA, Navarrete-Cortes J, Pulgar R, Santa S, Moya-Anegón F. World dental research production: An ISI database approach (19992003). Eur J Oral Sci, 2006; 114: 102-108.

6. Dorta-González P, Dorta-González MI. Central indexes to the citation distribution: A complement to the h-index. Scientometrics, 2011. En prensa. 7. Hirsch JE. An index to quantify an individual's scientific research output. PNAS, 2005; 102(46): 16569-16572.

8. Torres-Salinas D, López-Cozar ED, Jiménez-Contreras E. Ranking of departments and researchers within a university using two different databases: Web of Science versus Scopus. Scientometrics, 2009; 80(3): 761-774.

9. Testa J. Regional content expansion in web of science: Opening borders to exploration. Thomson-Reuters, 2010.

10. Arribalzaga E. Incorporación al ISI de la Revista Chilena de Cirugía. Rev Chil Cir, 2009; 61(4): 313.

11. Cofré J. Buenas noticias, ¡excelentes noticias para el mundo académico! Rev Chil Infect, 2008; 25 (3): 153.

12. Yang $\mathrm{S}$, Needleman $\mathrm{H}$, Niederman R. A bibliometric analysis of the pediatric dental literature in MEDLINE. Pediatr Dent, 2001; 23(5): 415-418. 13. Zhang YH, Pan YT, Hong X. A brief statistical analysis of stomatology papers published in international journals by researchers from the mainland of China-based on Thomson Reuters' journal citation reports for 2009. Int J Oral Sci, 2011; 3(1): 1-6.
14. Yuan JC, Lee DJ, Knoernschild KL, Campbell SD, Sukotjo C. Authorship characteristics in prosthodontic literature: Proliferation and internationalization. A review and analysis following a 10-year observation. J Prosthet Dent, 2010; 104(3): 158-164.

15. Assari S, Ahmadyar M. Dental research in Iran: A bibliometric analysis of electronically available literature. Int Dent J, 2009; 59(4): 210-214.

16. Kaur H, Gupta BM. Mapping of dental science research in India: A scientometric analysis of India's research output, 1999-2008. Scientometric, 2011; 85(1): 361-376.

17. Collet AM, Jara-Tracchia L, Palacios SB, Itoiz ME. Dental research productivity in Argentina (1993 to 2003). Acta Odontol Latinoam, 2006; 19(2): 81-84.

18. Ferraz VC, Amadei JR, Santos CF. The evolution of the Journal of Applied Oral Science: A bibliometric analysis. J Appl Oral Sci, 2008; 16(6): 420-427.

19. Cartes R. Scientific production of chilean dentistry in XXI century. XXIII Reunión Anual IADR División Chile. 7-9 de Octubre de 2010, Valdivia.

20. Bonitz M. Ten years Matthew effect for countries. Scientometrics, 2005; 64(3): 375-379.

21. Abramo G, Ciriaco A, Solazzo M. The relationship between scientists' research performance and the degree of internationalization of their research. Scientometrics, 2011; 86(3): 629-643.

22. Helene A, Ribeiro P. Brazilian scientific production, financial support, established investigators and doctoral graduates. Scientometrics, 2011. En prensa.

23. Lema F. La construcción de la sociedad del conocimiento en América Latina. La diáspora del conocimiento. Revista IDEA, 2004; 5. 